\title{
Some results on variational inequalities and generalized equilibrium problems with applications
}

\section{XIAOLONG QIN ${ }^{1}$, SUN YOUNG $\mathrm{CHO}^{2}$ and SHIN MIN $\mathrm{KANG}^{3}$}

${ }^{1}$ Department of Mathematics, Hangzhou Normal University, Hangzhou 310036, China

${ }^{2}$ Department of Mathematics, Gyeongsang National University, Jinju 660-701, Korea

${ }^{3}$ Department of Mathematics and the RINS, Gyeongsang National University Jinju 660-701, Korea

E-mail: smkang@gnu.ac.kr

\begin{abstract}
An iterative algorithm is considered for variational inequalities, generalized equilibrium problems and fixed point problems. Strong convergence of the proposed iterative algorithm is obtained in the framework Hilbert spaces.
\end{abstract}

Mathematical subject classification: $47 \mathrm{H} 05,47 \mathrm{H} 09,47 \mathrm{~J} 25,47 \mathrm{~N} 10$.

Key words: generalized equilibrium problem, variational inequality, fixed point, nonexpansive mapping.

\section{Introduction and preliminaries}

Let $H$ be a real Hilbert space, whose inner product and norm are denoted by $\langle\cdot, \cdot\rangle$ and $\|\cdot\|$, respectively. Let $C$ be a nonempty closed and convex subset of $H$ and $P_{C}$ be the projection of $H$ onto $C$.

Let $f, S, A, T$ be nonlinear mappings. Recall the following definitions:

(1) $f: C \rightarrow C$ is said to be $\alpha$-contractive if there exists a constant $\alpha \in(0,1)$ such that

$$
\|f x-f y\| \leq \alpha\|x-y\|, \quad \forall x, y \in C .
$$

\#CAM-90/09. Received: 08/IV/09. Accepted: 07/XII/09. 
(2) $S: C \rightarrow C$ is said to be nonexpansive if

$$
\|S x-S y\| \leq\|x-y\|, \quad \forall x, y \in C .
$$

Throughout this paper, we use $F(S)$ to denote the set of fixed points of the mapping $S$.

(3) $A: C \rightarrow H$ is said to be monotone if

$$
\langle A x-A y, x-y\rangle \geq 0, \quad \forall x, y \in C .
$$

(4) $A: C \rightarrow H$ is said to be inverse-strongly monotone if there exists $\delta>0$ such that

$$
\langle x-y, A x-A y\rangle \geq \delta\|A x-A y\|^{2}, \quad \forall x, y \in C .
$$

Such a mapping $A$ is also called $\delta$-inverse-strongly monotone. We know that if $S: C \rightarrow C$ is nonexpansive, then $A=I-S$ is $\frac{1}{2}$-inversestrongly monotone; see $[1,21]$ for more details.

(5) A set-valued mapping $T: H \rightarrow 2^{H}$ is said to be monotone if for all $x, y \in H, f \in T x$ and $g \in T y \Longrightarrow\langle x-y, f-g\rangle \geq 0$. A monotone mapping $T: H \rightarrow 2^{H}$ is maximal if the graph of $G(T)$ of $T$ is not properly contained in the graph of any other monotone mapping. It is known that a monotone mapping $T$ is maximal if and only if for $(x, f) \in$ $H \times H,\langle x-y, f-g\rangle \geq 0$ for every $(y, g) \in G(T)$ implies that $f \in T x$. Let $A$ be a monotone mapping of $C$ into $H$ and let $N_{C} v$ be the normal cone to $C$ at $v \in C$, i.e., $N_{C} v=\{w \in H:\langle v-u, w\rangle \geq 0, \forall u \in C\}$ and define

$$
T v= \begin{cases}A v+N_{C} v, & v \in C, \\ \emptyset, & v \notin C .\end{cases}
$$

Then $T$ is maximal monotone and $0 \in T v$ if and only if $\langle A v, u-v\rangle \geq 0$, $\forall u \in C$; see [16] for more details.

Recall that the classical variational inequality is to find an $x \in C$ such that

$$
\langle A x, y-x\rangle \geq 0, \quad \forall y \in C \text {. }
$$


In this paper, we use $\operatorname{VI}(C, A)$ to denote the solution set of the variational inequality (1.1). For given $z \in H$ and $u \in C$, we see that

$$
\langle u-z, v-u\rangle \geq 0, \quad \forall v \in C
$$

holds if and only if $u=P_{C} z$. It is known that projection operator $P_{C}$ satisfies

$$
\left\langle P_{C} x-P_{C} y, x-y\right\rangle \geq\left\|P_{C} x-P_{C} y\right\|^{2}, \quad \forall x, y \in H .
$$

One can see that the variational inequality (1.1) is equivalent to a fixed point problem. An element $u \in C$ is a solution of the variational inequality (1.1) if and only if $u \in C$ is a fixed point of the mapping $P_{C}(I-\lambda A) u$, where $\lambda>0$ is a constant and $I$ is the identity mapping. This can be seen from the following. $u \in C$ is a solution of the variational inequality (1.1), this is,

$$
\langle A u, y-u\rangle \geq 0, \quad \forall y \in C,
$$

which is equivalent to

$$
\langle(u-\lambda A u)-u, u-y\rangle \geq 0, \quad \forall y \in C,
$$

where $\lambda>0$ is a constant. This implies from (1.2) that $u=P_{C}(I-\lambda A) u$, that is, $u$ is a fixed point of the mapping $P_{C}(I-\lambda A)$. This alternative equivalent formulation has played a significant role in the studies of the variational inequalities and related optimization problems.

Let $A: C \rightarrow H$ be a $\delta$-inverse-strongly monotone mapping and $F$ be a bifunction of $C \times C$ into $\mathbb{R}$, where $\mathbb{R}$ denotes the set of real numbers. We consider the following generalized equilibrium problem:

Find $x \in C$ such that $F(x, y)+\langle A x, y-x\rangle \geq 0, \quad \forall y \in C$.

In this paper, the set of such an $x \in C$ is denoted by $E P(F, A)$, i.e.,

$$
E P(F, A)=\{x \in C: F(x, y)+\langle A x, y-x\rangle \geq 0, \forall y \in C\} .
$$

Next, we give some special cases of the generalized equilibrium problem (1.3).

(I) If $A \equiv 0$, the zero mapping, then the problem (1.3) is reduced to the following equilibrium problem:

$$
\text { Find } x \in C \text { such that } F(x, y) \geq 0, \quad \forall y \in C .
$$


In this paper, the set of such an $x \in C$ is denoted by $E P(F)$, i.e.,

$$
E P(F)=\{x \in C: F(x, y) \geq 0, \forall y \in C\} .
$$

(II) If $F \equiv 0$, then the problem (1.3) is reduced to the classical variational inequality (1.1).

In 2005, Iiduka and Takahashi [8] considered the classical variational inequality (1.1) and a single nonexpansive mapping. To be more precise, they obtained the following results.

Theorem IT. Let $C$ be a closed convex subset of a real Hilbert space $H$. Let $A$ be an $\alpha$-inverse-strongly monotone mapping of $C$ into $H$ and $S$ be a nonexpansive mapping of $C$ into itself such that $F(S) \cap V I(C, A) \neq \emptyset$. Suppose that $x_{1}=x \in C$ and $\left\{x_{n}\right\}$ is given by

$$
x_{n+1}=\alpha_{n} x+\left(1-\alpha_{n}\right) S P_{C}\left(x_{n}-\lambda_{n} A x_{n}\right), \quad \forall n \geq 1,
$$

where $\left\{\alpha_{n}\right\}$ is a sequence in $[0,1)$ and $\left\{\lambda_{n}\right\}$ is a sequence in $[0,2 \alpha]$. If $\left\{\alpha_{n}\right\}$ and $\left\{\lambda_{n}\right\}$ are chosen so that $\left\{\lambda_{n}\right\} \subset[a, b]$ for some $a, b$ with $0<a<b<2 \alpha$,

$$
\lim _{n \rightarrow \infty} \alpha_{n}=0, \sum_{n=1}^{\infty} \alpha_{n}=\infty, \sum_{n=1}^{\infty}\left|\alpha_{n+1}-\alpha_{n}\right|<\infty \text { and } \sum_{n=1}^{\infty}\left|\lambda_{n+1}-\lambda_{n}\right|<\infty,
$$

then $\left\{x_{n}\right\}$ converges strongly to $P_{F(S) \cap V I(C, A)} x$.

On the other hand, we see that the problem (1.3) is very general in the sense that it includes, as special cases, optimization problems, variational inequalities, mini-max problems, the Nash equilibrium problem in noncooperative games and others; see, for instance, $[2,5,9]$. Recently, many authors considered iterative methods for the problems (1.3) and (1.4), see [3-7, 11-15, 18, 20, 22, 24] for more details.

To study the equilibrium problems (1.3) and (1.4), we may assume that $F$ satisfies the following conditions:

(A1) $F(x, x)=0$ for all $x \in C$;

(A2) $F$ is monotone, i.e., $F(x, y)+F(y, x) \leq 0$ for all $x, y \in C$; 
(A3) for each $x, y, z \in C$,

$$
\limsup _{t \downarrow 0} F(t z+(1-t) x, y) \leq F(x, y) ;
$$

(A4) for each $x \in C, y \mapsto F(x, y)$ is convex and weakly lower semi-continuous.

Put $\mathcal{F}(x, y)=F(x, y)+\langle A x, y-x\rangle$ for each $x, y \in C$. It is not hard to see that $\mathcal{F}$ also confirms (A1)-(A4).

In 2007, Takahashi and Takahashi [20] introduced the following iterative method

$$
\left\{\begin{array}{l}
F\left(y_{n}, u\right)+\frac{1}{r_{n}}\left\langle u-y_{n}, y_{n}-x_{n}\right\rangle \geq 0, \quad \forall u \in C, \\
x_{n+1}=\alpha_{n} f\left(x_{n}\right)+\left(1-\alpha_{n}\right) T y_{n}, \quad n \geq 1,
\end{array}\right.
$$

where $f$ is a $\alpha$-contraction, $T$ is a nonexpansive mapping. They considered the problem of approximating a common element of the set of fixed points of a single nonexpansive mapping and the set of solutions of the equilibrium problem (1.4). Strong convergence theorems of the iterative algorithm (1.6) are established in a real Hilbert space.

Recently, Takahashi and Takahashi [22] further considered the generalized equilibrium problem (1.3). They obtained the following result in a real Hilbert space.

Theorem TT. Let $C$ be a closed convex subset of a real Hilbert space $H$ and $F: C \times C \rightarrow \mathbb{R}$ be a bi-function satisfying (A1)-(A4). Let $A$ be an $\alpha$-inversestrongly monotone mapping of $C$ into $H$ and $S$ be a non-expansive mapping of $C$ into itself such that $F(S) \cap E P(F, A) \neq \emptyset$. Let $u \in C$ and $x_{1} \in C$ and let $\left\{z_{n}\right\} \subset C$ and $\left\{x_{n}\right\} \subset C$ be sequences generated by

$$
\left\{\begin{array}{l}
F\left(z_{n}, y\right)+\left\langle A x_{n}, y-z_{n}\right\rangle+\frac{1}{r}\left\langle y-z_{n}, z_{n}-x_{n}\right\rangle \geq 0, \quad \forall y \in C, \\
x_{n+1}=\beta_{n} x_{n}+\left(1-\beta_{n}\right) S\left[\alpha_{n} u+\left(1-\alpha_{n}\right) z_{n}\right], \quad \forall n \geq 1,
\end{array}\right.
$$

where $\left\{\alpha_{n}\right\} \subset[0,1],\left\{\beta_{n}\right\} \subset[0,1]$ and $\left\{r_{n}\right\} \subset[0,2 \alpha]$ satisfy

$$
0<c \leq \beta_{n} \leq d<1, \quad 0<a \leq \lambda_{n} \leq b<2 \alpha,
$$




$$
\lim _{n \rightarrow \infty}\left(\lambda_{n}-\lambda_{n+1}\right)=0, \lim _{n \rightarrow \infty} \alpha_{n}=0, \text { and } \sum_{n=1}^{\infty} \alpha_{n}=\infty .
$$

Then, $\left\{x_{n}\right\}$ converges strongly to $z=P_{F(S) \cap E P(F, A)} u$.

Very recently, Chang, Lee and Chan [5] introduced a new iterative method for solving equilibrium problem (1.4), variational inequality (1.1) and the fixed point problem of nonexpansive mappings in the framework of Hilbert spaces. More precisely, they proved the following theorem.

Theorem CLC. Let H be a real Hilbert space, $C$ be a nonempty closed convex subset of $H$ and $F$ be a bifunction satisfying the conditions (A1)-(A4). Let $A: C \rightarrow H$ be an $\alpha$-inverse-strongly monotone mapping and $\left\{S_{i}: C \rightarrow C\right\}$ be a family of infinitely nonexpansive mappings with $F \cap V I(C, A) \cap E P(F) \neq \emptyset$, where $F:=\cap_{i=1}^{\infty} F\left(S_{i}\right)$ and $f: C \rightarrow C$ be a $\xi$-contractive mapping. Let $\left\{x_{n}\right\}$, $\left\{y_{n}\right\}\left\{k_{n}\right\}$ and $\left\{u_{n}\right\}$ be sequences defined by

$$
\left\{\begin{array}{l}
F\left(u_{n}, y\right)+\frac{1}{r_{n}}\left\langle y-u_{n}, u_{n}-x_{n}\right\rangle \geq 0, \quad \forall y \in C, \\
x_{n+1}=\alpha_{n} f\left(x_{n}\right)+\beta_{n} x_{n}+\gamma_{n} W_{n} k_{n}, \quad \forall n \geq 1, \\
k_{n}=P_{C}\left(y_{n}-\lambda_{n} A y_{n}\right), \\
y_{n}=P_{C}\left(u_{n}-\lambda_{n} A u_{n}\right),
\end{array}\right.
$$

where $\left\{W_{n}: C \rightarrow C\right\}$ is the sequence defined by (1.9), $\left\{\alpha_{n}\right\},\left\{\beta_{n}\right\}$ and $\left\{\gamma_{n}\right\}$ are sequences in $[0,1],\left\{\lambda_{n}\right\}$ is a sequence in $[a, b] \subset(0,2 \alpha)$ and $\left\{r_{n}\right\}$ is a sequence in $(0, \infty)$. If the following conditions are satisfied:

(1) $\alpha_{n}+\beta_{n}+\gamma_{n}=1$;

(2) $\lim _{n \rightarrow \infty} \alpha_{n}=0 ; \sum_{n=1}^{\infty} \alpha_{n}=\infty$;

(3) $0<\lim \inf _{n \rightarrow \infty} \beta_{n} \leq \lim \sup _{n \rightarrow \infty} \beta_{n}<1$;

(4) $\liminf _{n \rightarrow \infty} r_{n}>0 ; \sum_{n=1}^{\infty}\left|r_{n+1}-r_{n}\right|<\infty$;

(5) $\lim _{n \rightarrow \infty}\left|\lambda_{n+1}-\lambda_{n}\right|=0$,

then $\left\{x_{n}\right\}$ and $\left\{u_{n}\right\}$ converge strongly to $z \in F \cap V I(C, A) \cap E P(F)$. 
In this paper, motivated and inspired by the research going on in this direction, we introduce a general iterative method for finding a common element of the set of solutions of generalized equilibrium problems, the set of solutions of variational inequalities, and the set of common fixed points of a family of nonexpansive mappings in the framework of Hilbert spaces. The results presented in this paper improve and extend the corresponding results of Ceng and Yao [3, 4], Chang Lee and Chan [5], Iiduka and Takahashi [8], Qin, Shang and Zhou [12], Su, Shang and Qin [18], Takahashi and Takahashi [20, 22], Yao and Yao [25] and many others.

In order to prove our main results, we need the following definitions and lemmas.

A space $X$ is said to satisfy Opial condition [10] if for each sequence $\left\{x_{n}\right\}$ in $X$ which converges weakly to point $x \in X$, we have

$$
\liminf _{n \rightarrow \infty}\left\|x_{n}-x\right\|<\liminf _{n \rightarrow \infty}\left\|x_{n}-y\right\|, \quad \forall y \in X, y \neq x .
$$

Lemma 1.1 ([2]). Let $C$ be a nonempty closed convex subset of $H$ and $F$ : $C \times C \rightarrow \mathbb{R}$ be a bifunction satisfying (A1)-(A4). Then, for any $r>0$ and $x \in H$, there exists $z \in C$ such that

$$
F(z, y)+\frac{1}{r}\langle y-z, z-x\rangle \geq 0, \quad \forall y \in C .
$$

Lemma 1.2 ([2], [7]). Suppose that all the conditions in Lemma 1.1 are satisfied. For any give $r>0$ define a mapping $T_{r}: H \rightarrow C$ as follows:

$$
T_{r} x=\left\{z \in C: F(z, y)+\frac{1}{r}\langle y-z, z-x\rangle \geq 0, \forall y \in C\right\}, \forall x \in H,
$$

then the following conclusions hold:

(1) $T_{r}$ is single-valued;

(2) $T_{r}$ is firmly nonexpansive, i.e., for any $x, y \in H$,

$$
\left\|T_{r} x-T_{r} y\right\|^{2} \leq\left\langle T_{r} x-T_{r} y, x-y\right\rangle ;
$$

(3) $F\left(T_{r}\right)=E P(F)$;

(4) EP(F) is closed and convex. 
Lemma 1.3 ([23]). Assume that $\left\{\alpha_{n}\right\}$ is a sequence of nonnegative real numbers such that

$$
\alpha_{n+1} \leq\left(1-\gamma_{n}\right) \alpha_{n}+\delta_{n},
$$

where $\left\{\gamma_{n}\right\}$ is a sequence in $(0,1)$ and $\left\{\delta_{n}\right\}$ is a sequence such that

(1) $\sum_{n=1}^{\infty} \gamma_{n}=\infty$;

(2) $\lim \sup _{n \rightarrow \infty} \delta_{n} / \gamma_{n} \leq 0$ or $\sum_{n=1}^{\infty}\left|\delta_{n}\right|<\infty$.

Then $\lim _{n \rightarrow \infty} \alpha_{n}=0$.

Definition 1.4 ([19]). Let $\left\{S_{i}: C \rightarrow C\right\}$ be a family of infinitely nonexpansive mappings and $\left\{\gamma_{i}\right\}$ be a nonnegative real sequence with $0 \leq \gamma_{i}<1, \forall i \geq 1$. For $n \geq 1$ define a mapping $W_{n}: C \rightarrow C$ as follows:

$$
\begin{aligned}
& U_{n, n+1}=I, \\
& U_{n, n}=\gamma_{n} S_{n} U_{n, n+1}+\left(1-\gamma_{n}\right) I, \\
& U_{n, n-1}=\gamma_{n-1} S_{n-1} U_{n, n}+\left(1-\gamma_{n-1}\right) I, \\
& \vdots \\
& U_{n, k}=\gamma_{k} S_{k} U_{n, k+1}+\left(1-\gamma_{k}\right) I, \\
& U_{n, k-1}=\gamma_{k-1} S_{k-1} U_{n, k}+\left(1-\gamma_{k-1}\right) I, \\
& \vdots \\
& U_{n, 2}=\gamma_{2} S_{2} U_{n, 3}+\left(1-\gamma_{2}\right) I, \\
& W_{n}=U_{n, 1}=\gamma_{1} S_{1} U_{n, 2}+\left(1-\gamma_{1}\right) I .
\end{aligned}
$$

Such a mapping $W_{n}$ is nonexpansive from $C$ to $C$ and it is called a $W$-mapping generated by $S_{n}, S_{n-1}, \ldots, S_{1}$ and $\gamma_{n}, \gamma_{n-1}, \ldots, \gamma_{1}$.

Lemma 1.5 ([19]). Let $C$ be a nonempty closed convex subset of a Hilbert space $H,\left\{S_{i}: C \rightarrow C\right\}$ be a family of infinitely nonexpansive mappings with $\cap_{i=1}^{\infty} F\left(S_{i}\right) \neq \emptyset$ and $\left\{\gamma_{i}\right\}$ be a real sequence such that $0<\gamma_{i} \leq l<1, \forall i \geq 1$. Then

(1) $W_{n}$ is nonexpansive and $F\left(W_{n}\right)=\cap_{i=1}^{\infty} F\left(S_{i}\right)$, for each $n \geq 1$; 
(2) for each $x \in C$ and for each positive integer $k$, the limit $\lim _{n \rightarrow \infty} U_{n, k}$ exists.

(3) the mapping $W: C \rightarrow C$ defined by

$$
W x:=\lim _{n \rightarrow \infty} W_{n} x=\lim _{n \rightarrow \infty} U_{n, 1} x, \quad x \in C,
$$

is a nonexpansive mapping satisfying $F(W)=\cap_{i=1}^{\infty} F\left(S_{i}\right)$ and it is called the $W$-mapping generated by $S_{1}, S_{2}, \ldots$ and $\gamma_{1}, \gamma_{2}, \ldots$

Lemma 1.6 ([5]). Let $C$ be a nonempty closed convex subset of a Hilbert space $H,\left\{S_{i}: C \rightarrow C\right\}$ be a family of infinitely nonexpansive mappings with $\cap_{i=1}^{\infty} F\left(S_{i}\right) \neq \emptyset$ and $\left\{\gamma_{i}\right\}$ be a real sequence such that $0<\gamma_{i} \leq l<1, \forall i \geq 1$. If $K$ is any bounded subset of $C$, then

$$
\lim _{n \rightarrow \infty} \sup _{x \in K}\left\|W x-W_{n} x\right\|=0 .
$$

Throughout this paper, we always assume that $0<\gamma_{i} \leq l<1, \forall i \geq 1$.

Lemma 1.7 ([17]). Let $\left\{x_{n}\right\}$ and $\left\{y_{n}\right\}$ be bounded sequences in a Hilbert space $H$ and $\left\{\beta_{n}\right\}$ be a sequence in $[0,1]$ with

$$
0<\liminf _{n \rightarrow \infty} \beta_{n} \leq \limsup _{n \rightarrow \infty} \beta_{n}<1 .
$$

Suppose that $x_{n+1}=\left(1-\beta_{n}\right) y_{n}+\beta_{n} x_{n}$ for all $n \geq 0$ and

$$
\limsup _{n \rightarrow \infty}\left(\left\|y_{n+1}-y_{n}\right\|-\left\|x_{n+1}-x_{n}\right\|\right) \leq 0 .
$$

Then $\lim _{n \rightarrow \infty}\left\|y_{n}-x_{n}\right\|=0$.

\section{Main results}

Theorem 2.1. Let $C$ be a nonempty closed convex subset of a Hilbert space $H$ and $F$ be a bifunction from $C \times C$ to $\mathbb{R}$ which satisfies (A1)-(A4). Let $A_{1}: C \rightarrow$ $H$ be a $\delta_{1}$-inverse-strongly monotone mapping, $A_{2}: C \rightarrow H$ be a $\delta_{2}$-inversestrongly monotone mapping, $A_{3}: C \rightarrow H$ be a $\delta_{3}$-inverse-strongly monotone mapping and $\left\{S_{i}: C \rightarrow C\right\}$ be a family of infinitely nonexpansive mappings. 
Assume that $\Omega:=F P \cap E P\left(F, A_{3}\right) \cap V I \neq \emptyset$, where $F P=\cap_{i=1}^{\infty} F\left(S_{i}\right)$ and $V I=V I\left(C, A_{1}\right) \cap V I\left(C, A_{2}\right)$. Let $f: C \rightarrow C$ be an $\alpha$-contraction. Let $x_{1} \in C$ and $\left\{x_{n}\right\}$ be a sequence generated by

$$
\left\{\begin{array}{l}
F\left(u_{n}, y\right)+\left\langle A_{3} x_{n}, y-u_{n}\right\rangle+\frac{1}{r_{n}}\left\langle y-u_{n}, u_{n}-x_{n}\right\rangle \geq 0, \quad \forall y \in C, \\
z_{n}=P_{C}\left(u_{n}-\lambda_{n} A_{2} u_{n}\right), \\
y_{n}=P_{C}\left(z_{n}-\eta_{n} A_{1} z_{n}\right), \\
x_{n+1}=\alpha_{n} f\left(x_{n}\right)+\beta_{n} x_{n}+\gamma_{n} W_{n} y_{n}, \quad \forall n \geq 1,
\end{array}\right.
$$

where $\left\{W_{n}: C \rightarrow C\right\}$ is the sequence generated in (1.9), $\left\{\alpha_{n}\right\},\left\{\beta_{n}\right\}$ and $\left\{\gamma_{n}\right\}$ are sequences in $(0,1)$ such that $\alpha_{n}+\beta_{n}+\gamma_{n}=1$ for each $n \geq 1$ and $\left\{r_{n}\right\},\left\{\lambda_{n}\right\}$ and $\left\{\eta_{n}\right\}$ are positive number sequences. Assume that the above control sequences satisfy the following restrictions:

(R1) $0<a \leq \eta_{n} \leq b<2 \delta_{1}, 0<a^{\prime} \leq \lambda_{n} \leq b^{\prime}<2 \delta_{2}, 0<\bar{a} \leq r_{n} \leq \bar{b}<2 \delta_{3}$, $\forall n \geq 1$;

(R2) $\lim _{n \rightarrow \infty} \alpha_{n}=0$ and $\sum_{n=1}^{\infty} \alpha_{n}=\infty$;

(R3) $0<\liminf _{n \rightarrow \infty} \beta_{n} \leq \lim \sup _{n \rightarrow \infty} \beta_{n}<1$;

(R4) $\lim _{n \rightarrow \infty}\left(\lambda_{n}-\lambda_{n+1}\right)=\lim _{n \rightarrow \infty}\left(\eta_{n}-\eta_{n+1}\right)=\lim _{n \rightarrow \infty}\left(r_{n}-r_{n+1}\right)=0$.

Then the sequence $\left\{x_{n}\right\}$ converges strongly to $z \in \Omega$, which solves uniquely the following variational inequality:

$$
\langle(I-f) z, z-x\rangle \leq 0, \quad \forall x \in \Omega .
$$

Proof. First, we show, for each $n \geq 1$, that the mappings $I-\eta_{n} A_{1}, I-\lambda_{n} A_{2}$ and $I-r_{n} A_{3}$ are nonexpansive. Indeed, for $\forall x, y \in C$, we obtain from the restriction $(\mathrm{R} 1)$ that

$$
\begin{aligned}
& \left\|\left(I-\eta_{n} A_{1}\right) x-\left(I-\eta_{n} A_{1}\right) y\right\|^{2} \\
& =\left\|(x-y)-\eta_{n}\left(A_{1} x-A_{1} y\right)\right\|^{2} \\
& =\|x-y\|^{2}-2 \eta_{n}\left\langle x-y, A_{1} x-A_{1} y\right\rangle+\eta_{n}^{2}\left\|A_{1} x-A_{1} y\right\|^{2} \\
& \leq\|x-y\|^{2}-2 \eta_{n} \delta_{1}\left\|A_{1} x-A_{1} y\right\|^{2}+\eta_{n}^{2}\left\|A_{1} x-A_{1} y\right\|^{2} \\
& =\|x-y\|^{2}+\eta_{n}\left(\eta_{n}-2 \delta_{1}\right)\left\|A_{1} x-A_{1} y\right\|^{2} \\
& \leq\|x-y\|^{2}
\end{aligned}
$$


which implies that the mapping $I-\eta_{n} A_{1}$ is nonexpansive, so are $I-\lambda_{n} A_{2}$ and $I-r_{n} A_{3}$ for each $n \geq 1$. Note that $u_{n}$ can be re-written as $u_{n}=T_{r_{n}}(I-$ $\left.r_{n} A_{3}\right) x_{n}$ for each $n \geq 1$. Take $x^{*} \in \Omega$. Noticing that $x^{*}=P_{C}\left(I-\eta_{n} A_{1}\right) x^{*}=$ $P_{C}\left(I-\lambda_{n} A_{2}\right) x^{*}=T_{r_{n}}\left(I-r_{n} A_{3}\right) x^{*}$, we have

$$
\left\|u_{n}-x^{*}\right\|=\left\|T_{r_{n}}\left(I-r_{n} A_{3}\right) x_{n}-T_{r_{n}}\left(I-r_{n} A_{3}\right) x^{*}\right\| \leq\left\|x_{n}-x^{*}\right\| .
$$

On the other hand, we have

$$
\begin{aligned}
\left\|z_{n}-x^{*}\right\| & =\left\|P_{C}\left(u_{n}-\lambda_{n} A_{2} u_{n}\right)-P_{C}\left(x^{*}-\lambda_{n} A_{2} x^{*}\right)\right\| \\
& \leq\left\|\left(u_{n}-\lambda_{n} A_{2} u_{n}\right)-\left(x^{*}-\lambda_{n} A_{2} x^{*}\right)\right\| \\
& \leq\left\|u_{n}-x^{*}\right\| .
\end{aligned}
$$

It follows from (2.2) and (2.3) that

$$
\left\|z_{n}-x^{*}\right\| \leq\left\|x_{n}-x^{*}\right\|,
$$

which yields that

$$
\begin{aligned}
\left\|y_{n}-x^{*}\right\| & =\left\|P_{C}\left(z_{n}-\eta_{n} A_{1} z_{n}\right)-P_{C}\left(x^{*}-\eta_{n} A_{1} x^{*}\right)\right\| \\
& \leq\left\|\left(z_{n}-\eta_{n} A_{1} z_{n}\right)-\left(x^{*}-\eta_{n} A_{1} x^{*}\right)\right\| \\
& \leq\left\|z_{n}-x^{*}\right\| \\
& \leq\left\|x_{n}-x^{*}\right\| .
\end{aligned}
$$

From the algorithm (2.1) and (2.5), we arrive at

$$
\begin{aligned}
\left\|x_{n+1}-x^{*}\right\|= & \left\|\alpha_{n} f\left(x_{n}\right)+\beta_{n} x_{n}+\gamma_{n} W_{n} y_{n}-x^{*}\right\| \\
\leq & \alpha_{n}\left\|f\left(x_{n}\right)-x^{*}\right\|+\beta_{n}\left\|x_{n}-x^{*}\right\|+\gamma_{n}\left\|W_{n} y_{n}-x^{*}\right\| \\
\leq & \alpha_{n}\left\|f\left(x_{n}\right)-f\left(x^{*}\right)\right\|+\alpha_{n}\left\|f\left(x^{*}\right)-x^{*}\right\| \\
& +\beta_{n}\left\|x_{n}-x^{*}\right\|+\gamma_{n}\left\|y_{n}-x^{*}\right\| \\
\leq & \alpha \alpha_{n}\left\|x_{n}-x^{*}\right\|+\alpha_{n}\left\|f\left(x^{*}\right)-x^{*}\right\| \\
& +\beta_{n}\left\|x_{n}-x^{*}\right\|+\gamma_{n}\left\|x_{n}-x^{*}\right\| \\
= & {\left[1-\alpha_{n}(1-\alpha)\right]\left\|x_{n}-x^{*}\right\|+\alpha_{n}\left\|f\left(x^{*}\right)-x^{*}\right\| . }
\end{aligned}
$$


By simple inductions, we obtain that

$$
\left\|x_{n}-x^{*}\right\| \leq \max \left\{\left\|x_{1}-x^{*}\right\|, \frac{\left\|f\left(x^{*}\right)-x^{*}\right\|}{1-\alpha}\right\},
$$

which gives that the sequence $\left\{x_{n}\right\}$ is bounded, so are $\left\{y_{n}\right\},\left\{z_{n}\right\}$ and $\left\{u_{n}\right\}$. Without loss of generality, we can assume that there exists a bounded set $K \subset C$ such that

$$
x_{n}, y_{n}, z_{n}, u_{n} \in K, \quad \forall n \geq 1 .
$$

Notice that $u_{n+1}=T_{r_{n+1}}\left(I-r_{n+1} A_{3}\right) x_{n+1}$ and $u_{n}=T_{r_{n}}\left(I-r_{n} A_{3}\right) x_{n}$, we see from Lemma 1.2 that

$$
F\left(u_{n+1}, y\right)+\frac{1}{r_{n+1}}\left\langle y-u_{n+1}, u_{n+1}-\left(I-r_{n+1} A_{3}\right) x_{n+1}\right\rangle \geq 0, \quad \forall y \in C,
$$

and

$$
F\left(u_{n}, y\right)+\frac{1}{r_{n}}\left\langle y-u_{n}, u_{n}-\left(I-r_{n} A_{3}\right) x_{n}\right\rangle \geq 0, \quad \forall y \in C .
$$

Let $y=u_{n}$ in (2.7) and $y=u_{n+1}$ in (2.8). By adding up these two inequalities and using the assumption (R2), we obtain that

$$
\left\langle u_{n+1}-u_{n}, \frac{u_{n}-\left(I-r_{n} A_{3}\right) x_{n}}{r_{n}}-\frac{u_{n+1}-\left(I-r_{n+1} A_{3}\right) x_{n+1}}{r_{n+1}}\right\rangle \geq 0 .
$$

Hence, we have

$$
\begin{gathered}
\left\langle u_{n+1}-u_{n}, u_{n}-u_{n+1}+u_{n+1}-\left(I-r_{n} A_{3}\right) x_{n}\right. \\
\left.-\frac{r_{n}}{r_{n+1}}\left(u_{n+1}-\left(I-r_{n+1} A_{3}\right) x_{n+1}\right)\right\rangle \geq 0 .
\end{gathered}
$$

This implies that

$$
\begin{aligned}
\left\|u_{n+1}-u_{n}\right\|^{2} \leq & \left\langle u_{n+1}-u_{n},\left(I-r_{n+1} A_{3}\right) x_{n+1}-\left(I-r_{n} A_{3}\right) x_{n}\right. \\
& \left.+\left(1-\frac{r_{n}}{r_{n+1}}\right)\left(u_{n+1}-\left(I-r_{n+1} A_{3}\right) x_{n+1}\right)\right\rangle \\
\leq & \left\|u_{n+1}-u_{n}\right\|\left(\left\|\left(I-r_{n+1} A_{3}\right) x_{n+1}-\left(I-r_{n} A_{3}\right) x_{n}\right\|\right. \\
& \left.\left.+\left|1-\frac{r_{n}}{r_{n+1}}\right| \| u_{n+1}-\left(I-r_{n+1} A_{3}\right) x_{n+1}\right) \|\right) .
\end{aligned}
$$


It follows that

$$
\begin{aligned}
& \left\|u_{n+1}-u_{n}\right\| \\
& \leq\left\|\left(I-r_{n+1} A_{3}\right) x_{n+1}-\left(I-r_{n} A_{3}\right) x_{n}\right\| \\
& \quad+\frac{\left|r_{n+1}-r_{n}\right|}{r_{n+1}}\left\|u_{n+1}-\left(I-r_{n+1} A_{3}\right) x_{n+1}\right\| \\
& =\|\left(I-r_{n+1} A_{3}\right) x_{n+1}-\left(I-r_{n+1} A_{3}\right) x_{n} \\
& \quad+\left(I-r_{n+1} A_{3}\right) x_{n}-\left(I-r_{n} A_{3}\right) x_{n} \| \\
& \quad+\frac{\left|r_{n+1}-r_{n}\right|}{r_{n+1}}\left\|u_{n+1}-\left(I-r_{n+1} A_{3}\right) x_{n+1}\right\| \\
& \leq\left\|x_{n+1}-x_{n}\right\|+\left|r_{n+1}-r_{n}\right| M_{1},
\end{aligned}
$$

where $M_{1}$ is an appropriate constant such that

$$
M_{1}=\sup _{n \geq 1}\left\{\left\|A_{3} x_{n}\right\|+\frac{\left\|u_{n+1}-\left(I-r_{n+1} A_{3}\right) x_{n+1}\right\|}{\bar{a}}\right\} .
$$

From the nonexpansivity of $P_{C}$, we also have

$$
\begin{aligned}
& \left\|z_{n+1}-z_{n}\right\| \\
& =\left\|P_{C}\left(u_{n+1}-\lambda_{n+1} A_{2} u_{n+1}\right)-P_{C}\left(u_{n}-\lambda_{n} A_{2} u_{n}\right)\right\| \\
& \leq\left\|u_{n+1}-\lambda_{n+1} A_{2} u_{n+1}-\left(u_{n}-\lambda_{n} A_{2} u_{n}\right)\right\| \\
& =\left\|\left(I-\lambda_{n+1} A_{2}\right) u_{n+1}-\left(I-\lambda_{n+1} A_{2}\right) u_{n}+\left(\lambda_{n}-\lambda_{n+1}\right) A_{2} u_{n}\right\| \\
& \leq\left\|u_{n+1}-u_{n}\right\|+\left|\lambda_{n}-\lambda_{n+1}\right|\left\|A_{2} u_{n}\right\| .
\end{aligned}
$$

Substituting (2.9) into (2.10), we arrive at

$$
\left\|z_{n+1}-z_{n}\right\| \leq\left\|x_{n+1}-x_{n}\right\|+\left|r_{n+1}-r_{n}\right| M_{1}+\left|\lambda_{n}-\lambda_{n+1}\right|\left\|A_{2} u_{n}\right\| .
$$

In a similar way, we can obtain that

$$
\left\|y_{n+1}-y_{n}\right\| \leq\left\|z_{n+1}-z_{n}\right\|+\left|\eta_{n}-\eta_{n+1}\right|\left\|A_{1} z_{n}\right\| \text {. }
$$

Combining (2.11) with (2.12), we see that

$$
\left\|y_{n+1}-y_{n}\right\| \leq\left\|x_{n+1}-x_{n}\right\|+\left(\left|r_{n+1}-r_{n}\right|+\left|\lambda_{n}-\lambda_{n+1}\right|+\left|\eta_{n}-\eta_{n+1}\right|\right) M_{2} \text {, }
$$


where $M_{2}$ is an appropriate constant such that

$$
M_{2}=\max \left\{\sup _{n \geq 1}\left\{\left\|A_{1} z_{n}\right\|\right\}, \sup _{n \geq 1}\left\{\left\|A_{2} u_{n}\right\|\right\}, M_{1}\right\} .
$$

Letting

$$
x_{n+1}=\left(1-\beta_{n}\right) v_{n}+\beta_{n} x_{n}, \quad \forall n \geq 1,
$$

we see that

$$
\begin{aligned}
v_{n+1}-v_{n}= & \frac{\alpha_{n+1} f\left(x_{n+1}\right)+\gamma_{n+1} W_{n+1} y_{n+1}}{1-\beta_{n+1}}-\frac{\alpha_{n} f\left(x_{n}\right)+\gamma_{n} W_{n} y_{n}}{1-\beta_{n}} \\
= & \frac{\alpha_{n+1} f\left(x_{n+1}\right)}{1-\beta_{n+1}}+\frac{\left(1-\alpha_{n+1}-\beta_{n+1}\right) W_{n+1} y_{n+1}}{1-\beta_{n+1}} \\
& -\left(\frac{\alpha_{n} f\left(x_{n}\right)}{1-\beta_{n}}+\frac{\left(1-\alpha_{n}-\beta_{n}\right) W_{n} y_{n}}{1-\beta_{n}}\right) \\
= & \frac{\alpha_{n+1}}{1-\beta_{n+1}}\left(f\left(x_{n+1}\right)-W_{n+1} y_{n+1}\right)-\frac{\alpha_{n}}{1-\beta_{n}}\left(f\left(x_{n}\right)-W_{n} y_{n}\right) \\
& +W_{n+1} y_{n+1}-W_{n} y_{n} .
\end{aligned}
$$

It follows that

$$
\begin{aligned}
\left\|v_{n+1}-v_{n}\right\| \leq & \frac{\alpha_{n+1}}{1-\beta_{n+1}}\left\|f\left(x_{n+1}\right)-W_{n+1} y_{n+1}\right\| \\
& +\frac{\alpha_{n}}{1-\beta_{n}}\left\|f\left(x_{n}\right)-W_{n} y_{n}\right\|+\left\|W_{n+1} y_{n+1}-W_{n} y_{n}\right\| .
\end{aligned}
$$

On the other hand, we have

$$
\begin{aligned}
& \left\|W_{n+1} y_{n+1}-W_{n} y_{n}\right\| \\
& =\left\|W_{n+1} y_{n+1}-W y_{n+1}+W y_{n+1}-W y_{n}+W y_{n}-W_{n} y_{n}\right\| \\
& \leq\left\|W_{n+1} y_{n+1}-W y_{n+1}\right\|+\left\|W y_{n+1}-W y_{n}\right\|+\left\|W y_{n}-W_{n} y_{n}\right\| \\
& \leq \sup _{x \in K}\left\{\left\|W_{n+1} x-W x\right\|+\left\|W x-W_{n} x\right\|\right\}+\left\|y_{n+1}-y_{n}\right\|,
\end{aligned}
$$

where $K$ is the bounded subset of $C$ defined by (2.6). Substituting (2.13) into (2.16), we arrive at

$$
\begin{aligned}
\left\|W_{n+1} y_{n+1}-W_{n} y_{n}\right\| \leq & \sup _{x \in K}\left\{\left\|W_{n+1} x-W x\right\|+\mid W x-W_{n} x \|\right\}+\left\|x_{n+1}-x_{n}\right\| \\
& +\left(\left|r_{n+1}-r_{n}\right|+\left|\lambda_{n}-\lambda_{n+1}\right|+\left|\eta_{n}-\eta_{n+1}\right|\right) M_{2},
\end{aligned}
$$


which combines with (2.15) yields that

$$
\begin{aligned}
& \left\|v_{n+1}-v_{n}\right\|-\left\|x_{n+1}-x_{n}\right\| \\
& \leq \frac{\alpha_{n+1}}{1-\beta_{n+1}}\left\|f\left(x_{n+1}\right)-W_{n+1} y_{n+1}\right\|+\frac{\alpha_{n}}{1-\beta_{n}}\left\|f\left(x_{n}\right)-W_{n} y_{n}\right\| \\
& \quad+\sup _{x \in K}\left\{\left\|W_{n+1} x-W x\right\|+\left\|W x-W_{n} x\right\|\right\} \\
& \quad+\left(\left|r_{n+1}-r_{n}\right|+\left|\lambda_{n}-\lambda_{n+1}\right|+\left|\eta_{n}-\eta_{n+1}\right|\right) M_{2} .
\end{aligned}
$$

In view of the restriction (R2), (R3) and (R4), we obtain from Lemma 1.6 that

$$
\limsup _{n \rightarrow \infty}\left(\left\|v_{n+1}-v_{n}\right\|-\left\|x_{n+1}-x_{n}\right\|\right) \leq 0 .
$$

Hence, we obtain from Lemma 1.7 that

$$
\lim _{n \rightarrow \infty}\left\|v_{n}-x_{n}\right\|=0 .
$$

In view of (2.14), we have

$$
\left\|x_{n+1}-x_{n}\right\|=\left(1-\beta_{n}\right)\left\|v_{n}-x_{n}\right\| .
$$

Thanks to the restriction (R3), we see that

$$
\lim _{n \rightarrow \infty}\left\|x_{n+1}-x_{n}\right\|=0 .
$$

For any $x^{*} \in \Omega$, we see that

$$
\begin{aligned}
\left\|x_{n+1}-x^{*}\right\|^{2} & =\left\|\alpha_{n}\left(f\left(x_{n}\right)-x^{*}\right)+\beta_{n}\left(x_{n}-x^{*}\right)+\gamma_{n}\left(W_{n} y_{n}-x^{*}\right)\right\|^{2} \\
& \leq \alpha_{n}\left\|f\left(x_{n}\right)-x^{*}\right\|^{2}+\beta_{n}\left\|x_{n}-x^{*}\right\|^{2}+\gamma_{n}\left\|W_{n} y_{n}-x^{*}\right\|^{2} \\
& \leq \alpha_{n}\left\|f\left(x_{n}\right)-x^{*}\right\|^{2}+\beta_{n}\left\|x_{n}-x^{*}\right\|^{2}+\gamma_{n}\left\|y_{n}-x^{*}\right\|^{2} .
\end{aligned}
$$

Note that

$$
\begin{aligned}
\left\|y_{n}-x^{*}\right\|^{2}= & \left\|P_{C}\left(z_{n}-\eta_{n} A_{1} z_{n}\right)-x^{*}\right\|^{2} \\
\leq & \left\|\left(I-\eta_{n} A_{1}\right) z_{n}-\left(I-\eta_{n} A_{1}\right) x^{*}\right\|^{2} \\
= & \left\|\left(z_{n}-x^{*}\right)-\eta_{n}\left(A_{1} z_{n}-A_{1} x^{*}\right)\right\|^{2} \\
= & \left\|z_{n}-x^{*}\right\|^{2}-2 \eta_{n}\left\langle z_{n}-x^{*}, A_{1} z_{n}-A_{1} x^{*}\right\rangle \\
& +\eta_{n}^{2}\left\|A_{1} z_{n}-A_{1} x^{*}\right\|^{2} \\
\leq & \left\|z_{n}-x^{*}\right\|^{2}-2 \eta_{n} \delta_{1}\left\|A_{1} z_{n}-A_{1} x^{*}\right\|^{2} \\
& +\eta_{n}^{2}\left\|A_{1} z_{n}-A_{1} x^{*}\right\|^{2} \\
= & \left\|x_{n}-x^{*}\right\|^{2}+\eta_{n}\left(\eta_{n}-2 \delta_{1}\right)\left\|A_{1} z_{n}-A_{1} x^{*}\right\|^{2} .
\end{aligned}
$$


Substituting (2.19) into (2.18), we arrive at $\left\|x_{n+1}-x^{*}\right\|^{2} \leq \alpha_{n}\left\|f\left(x_{n}\right)-x^{*}\right\|^{2}+\left\|x_{n}-x^{*}\right\|^{2}+\gamma_{n} \eta_{n}\left(\eta_{n}-2 \delta_{1}\right)\left\|A_{1} z_{n}-A_{1} x^{*}\right\|^{2}$. This implies that

$$
\begin{aligned}
& \gamma_{n} \eta_{n}\left(2 \delta_{1}-\eta_{n}\right)\left\|A_{1} z_{n}-A_{1} x^{*}\right\|^{2} \\
& \leq \alpha_{n}\left\|f\left(x_{n}\right)-x^{*}\right\|^{2}+\left\|x_{n}-x^{*}\right\|^{2}-\left\|x_{n+1}-x^{*}\right\|^{2} \\
& \leq \alpha_{n}\left\|f\left(x_{n}\right)-x^{*}\right\|^{2}+\left(\left\|x_{n}-x^{*}\right\|+\left\|x_{n+1}-x^{*}\right\|\right)\left\|x_{n}-x_{n+1}\right\| .
\end{aligned}
$$

By virtue of the restrictions (R1) and (R2), we obtain from (2.17) that

$$
\lim _{n \rightarrow \infty}\left\|A_{1} z_{n}-A_{1} x^{*}\right\|=0 .
$$

Next, we show that

$$
\lim _{n \rightarrow \infty}\left\|A_{2} u_{n}-A_{2} x^{*}\right\|=0 .
$$

Indeed, by using (2.18), we obtain that

$$
\left\|x_{n+1}-x^{*}\right\|^{2} \leq \alpha_{n}\left\|f\left(x_{n}\right)-x^{*}\right\|^{2}+\beta_{n}\left\|x_{n}-x^{*}\right\|^{2}+\gamma_{n}\left\|z_{n}-x^{*}\right\|^{2} .
$$

On the other hand, we have

$$
\begin{aligned}
& \left\|z_{n}-x^{*}\right\|^{2}=\left\|P_{C}\left(u_{n}-\lambda_{n} A_{2} u_{n}\right)-x^{*}\right\|^{2} \\
& \leq\left\|\left(I-\lambda_{n} A_{2}\right) u_{n}-\left(I-\lambda_{n} A_{2}\right) x^{*}\right\|^{2} \\
& =\left\|\left(u_{n}-x^{*}\right)-\lambda_{n}\left(A_{2} u_{n}-A_{u} x^{*}\right)\right\|^{2} \\
& =\left\|u_{n}-x^{*}\right\|^{2}-2 \lambda_{n}\left\langle u_{n}-x^{*}, A_{2} u_{n}-A_{2} x^{*}\right\rangle+\lambda_{n}^{2}\left\|A_{2} u_{n}-A_{2} x^{*}\right\|^{2} \\
& \leq\left\|u_{n}-x^{*}\right\|^{2}-2 \lambda_{n} \delta_{2}\left\|A_{2} u_{n}-A_{2} x^{*}\right\|^{2}+\lambda_{n}^{2}\left\|A_{2} u_{n}-A_{2} x^{*}\right\|^{2} \\
& =\left\|u_{n}-x^{*}\right\|^{2}+\lambda_{n}\left(\lambda_{n}-2 \delta_{2}\right)\left\|A_{2} u_{n}-A_{2} x^{*}\right\|^{2} .
\end{aligned}
$$

Substituting (2.23) into (2.22), we arrive at

$$
\left\|x_{n+1}-x^{*}\right\|^{2} \leq \alpha_{n}\left\|f\left(x_{n}\right)-x^{*}\right\|^{2}+\left\|x_{n}-x^{*}\right\|^{2}+\gamma_{n} \lambda_{n}\left(\lambda_{n}-2 \delta_{2}\right)\left\|A_{2} u_{n}-A_{2} x^{*}\right\|^{2} .
$$

This in turn gives that

$$
\begin{aligned}
& \gamma_{n} \lambda_{n}\left(2 \delta_{2}-\lambda_{n}\right)\left\|A_{2} u_{n}-A_{2} x^{*}\right\|^{2} \\
& \leq \alpha_{n}\left\|f\left(x_{n}\right)-x^{*}\right\|^{2}+\left\|x_{n}-x^{*}\right\|^{2}-\left\|x_{n+1}-x^{*}\right\|^{2} \\
& \leq \alpha_{n}\left\|f\left(x_{n}\right)-x^{*}\right\|^{2}+\left(\left\|x_{n}-x^{*}\right\|+\left\|x_{n+1}-x^{*}\right\|\right)\left\|x_{n}-x_{n+1}\right\| .
\end{aligned}
$$


In view of the restrictions (R1) and (R2), we obtain from (2.17) that (2.21) holds.

On the other hand, we see from (2.22) that

$$
\left\|x_{n+1}-x^{*}\right\|^{2} \leq \alpha_{n}\left\|f\left(x_{n}\right)-x^{*}\right\|^{2}+\beta_{n}\left\|x_{n}-x^{*}\right\|^{2}+\gamma_{n}\left\|u_{n}-x^{*}\right\|^{2} .
$$

It follows that

$$
\begin{aligned}
\left\|x_{n+1}-x^{*}\right\|^{2} \leq & \alpha_{n}\left\|f\left(x_{n}\right)-x^{*}\right\|^{2}+\beta_{n}\left\|x_{n}-x^{*}\right\|^{2} \\
& +\gamma_{n}\left\|x_{n}-x^{*}-r_{n}\left(A_{3} x_{n}-A_{3} x^{*}\right)\right\|^{2} \\
\leq & \alpha_{n}\left\|f\left(x_{n}\right)-x^{*}\right\|^{2}+\beta_{n}\left\|x_{n}-x^{*}\right\|^{2} \\
& +\gamma_{n}\left(\left\|x_{n}-x^{*}\right\|^{2}+r_{n}^{2}\left\|A_{3} x_{n}-A_{3} x^{*}\right\|^{2}\right. \\
& \left.-2 r_{n}\left\langle A_{3} x_{n}-A_{3} x^{*}, x_{n}-x^{*}\right\rangle\right) \\
\leq & \alpha_{n}\left\|f\left(x_{n}\right)-x^{*}\right\|^{2}+\beta_{n}\left\|x_{n}-x^{*}\right\|^{2}+\gamma_{n}\left\|x_{n}-x^{*}\right\|^{2} \\
& -r_{n} \gamma_{n}\left(2 \delta_{3}-r_{n}\right)\left\|A_{3} x_{n}-A_{3} x^{*}\right\|^{2} .
\end{aligned}
$$

This implies that

$$
\begin{aligned}
& r_{n} \gamma_{n}\left(2 \delta_{3}-r_{n}\right)\left\|A_{3} x_{n}-A_{3} x^{*}\right\|^{2} \\
& \leq \alpha_{n}\left\|f\left(x_{n}\right)-x^{*}\right\|^{2}+\left\|x_{n}-x^{*}\right\|^{2}-\left\|x_{n+1}-x^{*}\right\|^{2} \\
& \leq \alpha_{n}\left\|f\left(x_{n}\right)-x^{*}\right\|^{2}+\left(\left\|x_{n}-x^{*}\right\|+\left\|x_{n+1}-x^{*}\right\|\right)\left\|x_{n}-x_{n+1}\right\| .
\end{aligned}
$$

In view of the restrictions (R1), (R2) and (R3), we see from (2.17) that

$$
\lim _{n \rightarrow \infty}\left\|A_{3} x_{n}-A_{3} x^{*}\right\|=0 .
$$

On the other hand, we see from Lemma 1.2 that

$$
\begin{aligned}
\left\|u_{n}-x^{*}\right\|^{2}= & \left\|T_{r_{n}}\left(I-r_{n} A_{3}\right) x_{n}-T_{r_{n}}\left(I-r_{n} A_{3}\right) x^{*}\right\|^{2} \\
\leq & \left\langle\left(I-r_{n} A_{3}\right) x_{n}-\left(I-r_{n} A_{3}\right) x^{*}, u_{n}-x^{*}\right\rangle \\
= & \frac{1}{2}\left(\left\|\left(I-r_{n} A_{3}\right) x_{n}-\left(I-r_{n} A_{3}\right) x^{*}\right\|^{2}+\left\|u_{n}-x^{*}\right\|^{2}\right. \\
& \left.-\left\|\left(I-r_{n} A_{3}\right) x_{n}-\left(I-r_{n} A_{3}\right) x^{*}-\left(u_{n}-x^{*}\right)\right\|^{2}\right) \\
\leq & \frac{1}{2}\left(\left\|x_{n}-x^{*}\right\|^{2}+\left\|u_{n}-x^{*}\right\|^{2}-\left\|x_{n}-u_{n}-r_{n}\left(A_{3} x_{n}-A_{3} x^{*}\right)\right\|^{2}\right) \\
= & \frac{1}{2}\left(\left\|x_{n}-x^{*}\right\|^{2}+\left\|u_{n}-x^{*}\right\|^{2}-\left\|x_{n}-u_{n}\right\|^{2}-r_{n}^{2}\left\|A_{3} x_{n}-A_{3} x^{*}\right\|^{2}\right. \\
& \left.+2 r_{n}\left\langle A_{3} x_{n}-A_{3} x^{*}, x_{n}-u_{n}\right\rangle\right) .
\end{aligned}
$$


This in turn implies that

$$
\begin{aligned}
\left\|u_{n}-x^{*}\right\|^{2} \leq & \left\|x_{n}-x^{*}\right\|^{2}-\left\|x_{n}-u_{n}\right\|^{2}-r_{n}^{2}\left\|A_{3} x_{n}-A_{3} x^{*}\right\|^{2} \\
& +2 r_{n}\left\langle A_{3} x_{n}-A_{3} x^{*}, x_{n}-u_{n}\right\rangle \\
\leq & \left\|x_{n}-x^{*}\right\|^{2}-\left\|x_{n}-u_{n}\right\|^{2} \\
& +2 r_{n}\left\|A_{3} x_{n}-A_{3} x^{*}\right\|\left\|x_{n}-u_{n}\right\| .
\end{aligned}
$$

Combining (2.24) with (2.26), we arrive at

$$
\begin{aligned}
\left\|x_{n+1}-x^{*}\right\|^{2} \leq & \alpha_{n}\left\|f\left(x_{n}\right)-x^{*}\right\|^{2}+\left\|x_{n}-x^{*}\right\|^{2}-\gamma_{n}\left\|x_{n}-u_{n}\right\|^{2} \\
& +2 r_{n}\left\|A_{3} x_{n}-A_{3} x^{*}\right\|\left\|x_{n}-u_{n}\right\| .
\end{aligned}
$$

It follows that

$$
\begin{aligned}
\gamma_{n}\left\|x_{n}-u_{n}\right\|^{2} \leq & \alpha_{n}\left\|f\left(x_{n}\right)-x^{*}\right\|^{2}+\left(\left\|x_{n}-x^{*}\right\|+\left\|x_{n+1}-x^{*}\right\|\right)\left\|x_{n}-x_{n+1}\right\| \\
& +2 r_{n}\left\|A_{3} x_{n}-A_{3} x^{*}\right\|\left\|x_{n}-u_{n}\right\| .
\end{aligned}
$$

Thanks to the restrictions (R2) and (R3), we see from (2.17) and (2.25) that

$$
\lim _{n \rightarrow \infty}\left\|x_{n}-u_{n}\right\|=0
$$

In view of the firm nonexpansivity of $P_{C}$, we see that

$$
\begin{aligned}
\left\|z_{n}-x^{*}\right\|^{2}= & \left\|P_{C}\left(I-\lambda_{n} A_{2}\right) u_{n}-P_{C}\left(I-\lambda_{n} A_{2}\right) x^{*}\right\|^{2} \\
\leq & \left\langle\left(I-\lambda_{n} A_{2}\right) u_{n}-\left(I-\lambda_{n} A_{2}\right) x^{*}, z_{n}-x^{*}\right\rangle \\
= & \frac{1}{2}\left(\left\|\left(I-\lambda_{n} A_{2}\right) u_{n}-\left(I-\lambda_{n} A_{2}\right) x^{*}\right\|^{2}+\left\|z_{n}-x^{*}\right\|^{2}\right. \\
& \left.-\left\|\left(I-\lambda_{n} A_{2}\right) u_{n}-\left(I-\lambda_{n} A_{2}\right) x^{*}-\left(z_{n}-x^{*}\right)\right\|^{2}\right) \\
\leq & \frac{1}{2}\left(\left\|u_{n}-x^{*}\right\|^{2}+\left\|z_{n}-x^{*}\right\|^{2}-\left\|u_{n}-z_{n}-\lambda_{n}\left(A_{2} u_{n}-A_{2} x^{*}\right)\right\|^{2}\right) \\
= & \frac{1}{2}\left(\left\|u_{n}-x^{*}\right\|^{2}+\left\|z_{n}-x^{*}\right\|^{2}-\left\|u_{n}-z_{n}\right\|^{2}\right. \\
& \left.+2 \lambda_{n}\left\langle u_{n}-z_{n}, A_{2} u_{n}-A_{2} x^{*}\right\rangle-\lambda_{n}^{2}\left\|A_{2} u_{n}-A_{2} x^{*}\right\|^{2}\right),
\end{aligned}
$$

which implies that

$$
\begin{aligned}
& \left\|z_{n}-x^{*}\right\|^{2} \leq\left\|u_{n}-x^{*}\right\|^{2}-\left\|u_{n}-z_{n}\right\|^{2} \\
& \quad+2 \lambda_{n}\left\langle u_{n}-z_{n}, A_{2} u_{n}-A_{2} x^{*}\right\rangle-\lambda_{n}^{2}\left\|A_{2} u_{n}-A_{2} x^{*}\right\|^{2} \\
& \leq\left\|x_{n}-x^{*}\right\|^{2}-\left\|u_{n}-z_{n}\right\|^{2}+2 \lambda_{n}\left\|u_{n}-z_{n}\right\|\left\|A_{2} u_{n}-A_{2} x^{*}\right\| .
\end{aligned}
$$


Substituting (2.28) into (2.22), we arrive at

$$
\begin{aligned}
\left\|x_{n+1}-x^{*}\right\|^{2} \leq & \alpha_{n}\left\|f\left(x_{n}\right)-x^{*}\right\|^{2}+\left\|x_{n}-x^{*}\right\|^{2}-\gamma_{n}\left\|u_{n}-z_{n}\right\|^{2} \\
& +2 \lambda_{n}\left\|u_{n}-z_{n}\right\|\left\|A_{2} u_{n}-A_{2} x^{*}\right\|,
\end{aligned}
$$

from which it follows that

$$
\begin{aligned}
\gamma_{n}\left\|u_{n}-z_{n}\right\|^{2} \leq & \alpha_{n}\left\|f\left(x_{n}\right)-x^{*}\right\|^{2}+\left\|x_{n}-x^{*}\right\|^{2}-\left\|x_{n+1}-x^{*}\right\|^{2} \\
& +2 \lambda_{n}\left\|u_{n}-z_{n}\right\|\left\|A_{2} u_{n}-A_{2} x^{*}\right\| \\
\leq & \alpha_{n}\left\|f\left(x_{n}\right)-x^{*}\right\|^{2}+\left(\left\|x_{n}-x^{*}\right\|+\left\|x_{n+1}-x^{*}\right\|\right)\left\|x_{n}-x_{n+1}\right\| \\
& +2 \lambda_{n}\left\|u_{n}-z_{n}\right\|\left\|A_{2} u_{n}-A_{2} x^{*}\right\| .
\end{aligned}
$$

In view of the restrictions (R2) and (R3), we obtain from (2.17) and (2.21) that

$$
\lim _{n \rightarrow \infty}\left\|u_{n}-z_{n}\right\|=0 .
$$

In a similar way, we can obtain that

$$
\lim _{n \rightarrow \infty}\left\|y_{n}-z_{n}\right\|=0
$$

Note that

$$
\begin{aligned}
\left\|W_{n} y_{n}-x_{n}\right\| & \leq\left\|x_{n}-x_{n+1}\right\|+\left\|x_{n+1}-W_{n} y_{n}\right\| \\
& \leq\left\|x_{n}-x_{n+1}\right\|+\alpha_{n}\left\|f\left(x_{n}\right)-W_{n} y_{n}\right\|+\beta_{n}\left\|x_{n}-W_{n} y_{n}\right\| .
\end{aligned}
$$

It follows that

$$
\left(1-\beta_{n}\right)\left\|W_{n} y_{n}-x_{n}\right\| \leq\left\|x_{n}-x_{n+1}\right\|+\alpha_{n}\left\|f\left(x_{n}\right)-W_{n} y_{n}\right\| .
$$

In view of the restrictions (R2) and (R3), we obtain from (2.17) that

$$
\lim _{n \rightarrow \infty}\left\|W_{n} y_{n}-x_{n}\right\|=0 .
$$

Notice that

$$
\left\|W_{n} y_{n}-y_{n}\right\| \leq\left\|y_{n}-z_{n}\right\|+\left\|z_{n}-u_{n}\right\|+\left\|u_{n}-x_{n}\right\|+\left\|x_{n}-W_{n} y_{n}\right\| .
$$

From (2.27), (2.29), (2.30) and (2.31), we arrive at

$$
\lim _{n \rightarrow \infty}\left\|W_{n} y_{n}-y_{n}\right\|=0 .
$$


Next, we prove that

$$
\limsup _{n \rightarrow \infty}\left\langle(f-I) z, x_{n}-z\right\rangle \leq 0,
$$

where $z=P_{\Omega} f(z)$. To see this, we choose a subsequence $\left\{x_{n_{i}}\right\}$ of $\left\{x_{n}\right\}$ such that

$$
\limsup _{n \rightarrow \infty}\left\langle(f-I) z, x_{n}-z\right\rangle=\lim _{i \rightarrow \infty}\left\langle(f-I) z, x_{n_{i}}-z\right\rangle .
$$

Since $\left\{x_{n_{i}}\right\}$ is bounded, there exists a subsequence $\left\{x_{n_{i_{j}}}\right\}$ of $\left\{x_{n_{i}}\right\}$ which converges weakly to $w$. Without loss of generality, we may assume that $x_{n_{i}} \rightarrow w$. On the other hand, we have

$$
\left\|x_{n}-y_{n}\right\| \leq\left\|x_{n}-u_{n}\right\|+\left\|u_{n}-z_{n}\right\|+\left\|z_{n}-y_{n}\right\| .
$$

It follows from (2.27), (2.29) and (2.30) that

$$
\lim _{n \rightarrow \infty}\left\|x_{n}-y_{n}\right\|=0 .
$$

Therefore, we see that $y_{n_{i}} \rightarrow w$. First, we prove that $w \in V I\left(C, A_{1}\right)$. For the purpose, let $T$ be the maximal monotone mapping defined by:

$$
T x= \begin{cases}A_{1} x+N_{C} x, & x \in C, \\ \emptyset, & x \notin C .\end{cases}
$$

For any given $(x, y) \in G(T)$, hence $y-A_{1} x \in N_{C}$. Since $y_{n} \in C$, by the definition of $N_{C}$, we have

$$
\left\langle x-y_{n}, y-A_{1} x\right\rangle \geq 0 .
$$

Notice that

$$
y_{n}=P_{C}\left(I-\eta_{n} A_{1}\right) z_{n} .
$$

It follows that

$$
\left\langle x-y_{n}, y_{n}-\left(I-\eta_{n} A_{1}\right) z_{n}\right\rangle \geq 0
$$

and hence

$$
\left\langle x-y_{n}, \frac{y_{n}-z_{n}}{\eta_{n}}+A_{1} z_{n}\right\rangle \geq 0 .
$$


From the monotonicity of $A_{1}$, we see that

$$
\begin{aligned}
\left\langle x-y_{n_{i}}, y\right\rangle \geq & \left\langle x-y_{n_{i}}, A_{1} x\right\rangle \\
\geq & \left\langle x-y_{n_{i}}, A_{1} x\right\rangle-\left\langle x-y_{n_{i}}, \frac{y_{n_{i}}-z_{n_{i}}}{\eta_{n_{i}}}+A_{1} z_{n_{i}}\right\rangle \\
= & \left\langle x-y_{n_{i}}, A_{1} x-A_{1} y_{n_{i}}\right\rangle+\left\langle x-y_{n_{i}}, A_{1} y_{n_{i}}-A_{1} z_{n_{i}}\right\rangle \\
& -\left\langle x-y_{n_{i}}, \frac{y_{n_{i}}-z_{n_{i}}}{\eta_{n_{i}}}\right\rangle \\
\geq & \left\langle x-y_{n_{i}}, A_{1} y_{n_{i}}-A_{1} z_{n_{i}}\right\rangle-\left\langle x-y_{n_{i}}, \frac{y_{n_{i}}-z_{n_{i}}}{\eta_{n_{i}}}\right\rangle .
\end{aligned}
$$

Since $y_{n_{i}} \rightarrow w$ and $A_{1}$ is Lipschitz continuous, we obtain from (2.30) that $\langle x-w, y\rangle \geq 0$. Notice that $T$ is maximal monotone, hence $0 \in T w$. This shows that $w \in V I\left(C, A_{1}\right)$. It follows from (2.27) and (2.29), we also have

$$
\lim _{n \rightarrow \infty}\left\|x_{n}-z_{n}\right\|=0
$$

Therefore, we obtain $z_{n_{i}} \rightarrow w$. Similarly, we can prove $w \in V I\left(C, A_{2}\right)$. That is, $w \in V I=V I\left(C, A_{2}\right) \cap V I\left(C, A_{1}\right)$.

Next, we show that $w \in F P=\cap_{i=1}^{\infty} F\left(S_{i}\right)$. Suppose the contrary, $w \notin F P$, i.e., $W w \neq w$. Since $y_{n_{i}} \rightarrow w$, we see from Opial condition that

$$
\begin{aligned}
\liminf _{i \rightarrow \infty}\left\|y_{n_{i}}-w\right\| & <\liminf _{i \rightarrow \infty}\left\|y_{n_{i}}-W w\right\| \\
& \leq \liminf _{i \rightarrow \infty}\left\{\left\|y_{n_{i}}-W y_{n_{i}}\right\|+\left\|W y_{n_{i}}-W w\right\|\right\} \\
& \leq \liminf _{i \rightarrow \infty}\left\{\left\|y_{n_{i}}-W y_{n_{i}}\right\|+\left\|y_{n_{i}}-w\right\|\right\} .
\end{aligned}
$$

On the other hand, we have

$$
\begin{aligned}
\left\|W y_{n}-y_{n}\right\| & \leq\left\|W y_{n}-W_{n} y_{n}\right\|+\left\|W_{n} y_{n}-y_{n}\right\| \\
& \leq \sup _{x \in K}\left\|W x-W_{n} x\right\|+\left\|W_{n} y_{n}-y_{n}\right\| .
\end{aligned}
$$

From Lemma 1.6, we obtain from (2.32) that $\lim _{n \rightarrow \infty}\left\|W y_{n}-y_{n}\right\|=0$, which combines with (2.36) yields that that

$$
\liminf _{i \rightarrow \infty}\left\|y_{n_{i}}-w\right\|<\liminf _{i \rightarrow \infty}\left\|y_{n_{i}}-w\right\| .
$$


This derives a contradiction. Thus, we have $w \in F P$.

Next, we show that $w \in E P\left(F, A_{3}\right)$. It follows from (2.27) that $u_{n} \rightarrow w$. Since $u_{n}=T_{r_{n}}\left(I-r A_{3}\right) x_{n}$, for any $y \in C$, we have

$$
F\left(u_{n}, y\right)+\left\langle A_{3} x_{n}, y-u_{n}\right\rangle+\frac{1}{r_{n}}\left\langle y-u_{n}, u_{n}-x_{n}\right\rangle \geq 0 .
$$

From the assumption (A2), we see that

$$
\left\langle A_{3} x_{n}, y-u_{n}\right\rangle+\frac{1}{r_{n}}\left\langle y-u_{n}, u_{n}-x_{n}\right\rangle \geq F\left(y, u_{n}\right), \quad \forall y \in C .
$$

Replacing $n$ by $n_{i}$, we arrive at

$$
\left\langle A_{3} x_{n_{i}}, y-u_{n_{i}}\right\rangle+\left\langle y-u_{n_{i}}, \frac{u_{n_{i}}-x_{n_{i}}}{r_{n_{i}}}\right\rangle \geq F\left(y, u_{n_{i}}\right), \quad \forall y \in C .
$$

Putting $y_{t}=t y+(1-t) w$ for any $t \in(0,1]$ and $y \in C$, we see that $y_{t} \in C$. It follows from (2.37) that

$$
\begin{aligned}
\left\langle y_{t}-u_{n_{i}}, A_{3} y_{t}\right\rangle \geq & \left\langle y_{t}-u_{n_{i}}, A_{3} y_{t}\right\rangle-\left\langle A_{3} x_{n_{i}}, y_{t}-u_{n_{i}}\right\rangle \\
& -\left\langle y_{t}-u_{n_{i}}, \frac{u_{n_{i}}-x_{n_{i}}}{r_{n_{i}}}\right\rangle+F\left(y_{t}, u_{n_{i}}\right) \\
= & \left\langle y_{t}-u_{n_{i}}, A_{3} y_{t}-A_{3} u_{n_{i}}\right\rangle+\left\langle y_{t}-u_{n_{i}}, A_{3} u_{n_{i}}-A_{3} x_{n_{i}}\right\rangle \\
& -\left\langle y_{t}-u_{n_{i}}, \frac{u_{n_{i}}-x_{n_{i}}}{r_{n_{i}}}\right\rangle+F\left(y_{t}, u_{n_{i}}\right) .
\end{aligned}
$$

In view of the monotonicity of $A_{3},(2.27)$ and the restriction (R1), we obtain from the assumption (A4) that

$$
\left\langle y_{t}-w, A_{3} y_{t}\right\rangle \geq F\left(y_{t}, w\right) .
$$

From the assumptions (A1) and (A4), we see that

$$
\begin{aligned}
0 & =F\left(y_{t}, y_{t}\right) \leq t F\left(y_{t}, y\right)+(1-t) F\left(y_{t}, w\right) \\
& \leq t F\left(y_{t}, y\right)+(1-t)\left\langle y_{t}-w, A_{3} y_{t}\right\rangle \\
& =t F\left(y_{t}, y\right)+(1-t) t\left\langle y-w, A_{3} y_{t}\right\rangle,
\end{aligned}
$$

from which it follows that

$$
0 \leq F\left(y_{t}, y\right)+(1-t)\left\langle y-w, A_{3} y_{t}\right\rangle, \quad \forall y \in C .
$$


It follows from the assumption (A3) that $w \in E P\left(F, A_{3}\right)$. On the other hand, we see from (2.33) that

$$
\limsup _{n \rightarrow \infty}\left\langle(f-I) z, x_{n}-z\right\rangle=\langle(f-I) z, w-z\rangle \leq 0 .
$$

Finally, we show that $x_{n} \rightarrow z$, as $n \rightarrow \infty$. Note that

$$
\begin{aligned}
&\left\|x_{n+1}-z\right\|^{2}=\left\langle\alpha_{n} f\left(x_{n}\right)+\beta_{n} x_{n}+\gamma_{n} W_{n} y_{n}-z, x_{n+1}-z\right\rangle \\
&=\alpha_{n}\left\langle f\left(x_{n}\right)-z, x_{n+1}-z\right\rangle+\beta_{n}\left\langle x_{n}-z, x_{n+1}-z\right\rangle \\
& \quad+\gamma_{n}\left\langle W_{n} y_{n}-z, x_{n+1}-z\right\rangle \\
& \leq \alpha_{n}\left\langle f\left(x_{n}\right)-f(z), x_{n+1}-z\right\rangle+\alpha_{n}\left\langle f(z)-z, x_{n+1}-z\right\rangle \\
&+\beta_{n}\left\|x_{n}-z\right\|\left\|x_{n+1}-z\right\|+\gamma_{n}\left\|y_{n}-z\right\|\left\|x_{n+1}-z\right\| \\
& \leq \frac{\alpha}{2} \alpha_{n}\left(\left\|x_{n}-z\right\|^{2}+\left\|x_{n+1}-z\right\|^{2}\right)+\alpha_{n}\left\langle f(z)-z, x_{n+1}-z\right\rangle \\
&+\left(1-\alpha_{n}\right)\left\|x_{n}-z\right\|\left\|x_{n+1}-z\right\| \\
& \leq \frac{\alpha}{2} \alpha_{n}\left(\left\|x_{n}-z\right\|^{2}+\left\|x_{n+1}-z\right\|^{2}\right)+\alpha_{n}\left\langle f(z)-z, x_{n+1}-z\right\rangle \\
&+\frac{\left(1-\alpha_{n}\right)}{2}\left(\left\|x_{n}-z\right\|^{2}+\left\|x_{n+1}-z\right\|^{2}\right) \\
& \leq \frac{1-\alpha_{n}(1-\alpha)}{2}\left\|x_{n}-z\right\|^{2}+\frac{1}{2}\left\|x_{n+1}-z\right\|^{2}+\alpha_{n}\left\langle f(z)-z, x_{n+1}-z\right\rangle,
\end{aligned}
$$

which implies that

$$
\left\|x_{n+1}-z\right\|^{2} \leq\left[1-\alpha_{n}(1-\alpha)\right]\left\|x_{n}-z\right\|^{2}+2 \alpha_{n}\left\langle f(z)-z, x_{n+1}-z\right\rangle .
$$

From the restriction (R2), we obtain from Lemma 1.3 that $\lim _{n \rightarrow \infty}\left\|x_{n}-z\right\|=$ 0 . This completes the proof.

Corollary 2.2. Let $C$ be a nonempty closed convex subset of a Hilbert space $H$ and $F$ be a bifunction from $C \times C$ to $\mathbb{R}$ which satisfies (A1)-(A4). Let $A_{1}$ : $C \rightarrow H$ be a $\delta_{1}$-inverse-strongly monotone mapping, $A_{2}: C \rightarrow H$ be a $\delta_{2}$ inverse-strongly monotone mapping and $\left\{S_{i}: C \rightarrow C\right\}$ be a family of infinitely nonexpansive mappings. Assume that $\Omega:=F P \cap E P(F) \cap V I \neq \emptyset$, where 
$F P=\cap_{i=1}^{\infty} F\left(S_{i}\right)$ and $V I=V I\left(C, A_{1}\right) \cap V I\left(C, A_{2}\right)$. Let $f: C \rightarrow C$ be an $\alpha$-contraction. Let $x_{1} \in C$ and $\left\{x_{n}\right\}$ be a sequence generated by

$$
\left\{\begin{array}{l}
F\left(u_{n}, y\right)+\frac{1}{r_{n}}\left\langle y-u_{n}, u_{n}-x_{n}\right\rangle \geq 0, \quad \forall y \in C, \\
z_{n}=P_{C}\left(u_{n}-\lambda_{n} A_{2} u_{n}\right), \\
y_{n}=P_{C}\left(z_{n}-\eta_{n} A_{1} z_{n}\right), \\
x_{n+1}=\alpha_{n} f\left(x_{n}\right)+\beta_{n} x_{n}+\gamma_{n} W_{n} y_{n}, \quad \forall n \geq 1,
\end{array}\right.
$$

where $\left\{W_{n}: C \rightarrow C\right\}$ is the sequence generated in (1.9), $\left\{\alpha_{n}\right\},\left\{\beta_{n}\right\}$ and $\left\{\gamma_{n}\right\}$ are sequences in $(0,1)$ such that $\alpha_{n}+\beta_{n}+\gamma_{n}=1$ for each $n \geq 1$ and $\left\{r_{n}\right\},\left\{\lambda_{n}\right\}$ and $\left\{\eta_{n}\right\}$ are positive number sequences. Assume that the above control sequences satisfy the following restrictions:

(R1) $0<a \leq \eta_{n} \leq b<2 \delta_{1}, 0<a^{\prime} \leq \lambda_{n} \leq b^{\prime}<2 \delta_{2}, 0<\bar{a} \leq r_{n} \leq \bar{b}<2 \delta_{3}$, $\forall n \geq 1$;

(R2) $\lim _{n \rightarrow \infty} \alpha_{n}=0$ and $\sum_{n=1}^{\infty} \alpha_{n}=\infty$;

(R3) $0<\liminf \operatorname{in}_{n \rightarrow \infty} \beta_{n} \leq \lim \sup _{n \rightarrow \infty} \beta_{n}<1$;

(R4) $\lim _{n \rightarrow \infty}\left(\lambda_{n}-\lambda_{n+1}\right)=\lim _{n \rightarrow \infty}\left(\eta_{n}-\eta_{n+1}\right)=\lim _{n \rightarrow \infty}\left(r_{n}-r_{n+1}\right)=0$.

Then the sequence $\left\{x_{n}\right\}$ converges strongly to $z \in \Omega$, which solves uniquely the following variational inequality:

$$
\langle(I-f) z, z-x\rangle \leq 0, \quad \forall x \in \Omega .
$$

Proof. Putting $A_{3} \equiv 0$, we see that

$$
\left\langle A_{3} x-A_{3} y, x-y\right\rangle \geq \delta\left\|A_{3} x-A_{3} y\right\|^{2}, \quad \forall x, y \in C
$$

for all $\delta \in(0, \infty)$. We can conclude from Theorem 2.1 the desired conclusion easily. This completes the proof.

Remark 2.3. If $A_{1} \equiv A_{2}$ and $\lambda_{n} \equiv \eta_{n}$, then Corollary 2.2 is reduced to Theorem 3.1 of Chang et al. [5]. If $A_{2} \equiv 0, f(x) \equiv e \in C$ a arbitrary fixed point and $S_{i} \equiv I$, the identity mapping, then Corollary 2.2 is reduced to Theorem 3.1 of Plubtieng and Punpaeng [11]. 
Corollary 2.4. Let $C$ be a nonempty closed convex subset of a Hilbert space $H$. Let $A_{1}: C \rightarrow H$ be a $\delta_{1}$-inverse-strongly monotone mapping, $A_{2}: C \rightarrow H$ be a $\delta_{2}$-inverse-strongly monotone mapping, $A_{3}: C \rightarrow H$ be a $\delta_{3}$-inverse-strongly monotone mapping and $\left\{S_{i}: C \rightarrow C\right\}$ be a family of infinitely nonexpansive mappings. Assume that $\Omega:=F P \cap E P\left(F, A_{3}\right) \cap V I \neq \emptyset$, where $F P=$ $\cap_{i=1}^{\infty} F\left(S_{i}\right)$ and $V I=V I\left(C, A_{1}\right) \cap V I\left(C, A_{2}\right)$. Let $f: C \rightarrow C$ be an $\alpha$ contraction. Let $x_{1} \in C$ and $\left\{x_{n}\right\}$ be a sequence generated by

$$
\left\{\begin{array}{l}
z_{n}=P_{C}\left(x_{n}-r_{n} A_{3} x_{n}\right), \\
z_{n}=P_{C}\left(u_{n}-\lambda_{n} A_{2} u_{n}\right), \\
y_{n}=P_{C}\left(z_{n}-\eta_{n} A_{1} z_{n}\right), \\
x_{n+1}=\alpha_{n} f\left(x_{n}\right)+\beta_{n} x_{n}+\gamma_{n} W_{n} y_{n}, \quad \forall n \geq 1,
\end{array}\right.
$$

where $\left\{W_{n}: C \rightarrow C\right\}$ is the sequence generated in (1.9), $\left\{\alpha_{n}\right\},\left\{\beta_{n}\right\}$ and $\left\{\gamma_{n}\right\}$ are sequences in $(0,1)$ such that $\alpha_{n}+\beta_{n}+\gamma_{n}=1$ for each $n \geq 1$ and $\left\{r_{n}\right\},\left\{\lambda_{n}\right\}$ and $\left\{\eta_{n}\right\}$ are positive number sequences. Assume that the above control sequences satisfy the following restrictions:

(R1) $0<a \leq \eta_{n} \leq b<2 \delta_{1}, 0<a^{\prime} \leq \lambda_{n} \leq b^{\prime}<2 \delta_{2}, 0<\bar{a} \leq r_{n} \leq \bar{b}<2 \delta_{3}$, $\forall n \geq 1$

(R2) $\lim _{n \rightarrow \infty} \alpha_{n}=0$ and $\sum_{n=1}^{\infty} \alpha_{n}=\infty$;

(R3) $0<\liminf _{n \rightarrow \infty} \beta_{n} \leq \lim \sup _{n \rightarrow \infty} \beta_{n}<1$;

(R4) $\lim _{n \rightarrow \infty}\left(\lambda_{n}-\lambda_{n+1}\right)=\lim _{n \rightarrow \infty}\left(\eta_{n}-\eta_{n+1}\right)=\lim _{n \rightarrow \infty}\left(r_{n}-r_{n+1}\right)=0$.

Then the sequence $\left\{x_{n}\right\}$ converges strongly to $z \in \Omega$, which solves uniquely the following variational inequality:

$$
\langle(I-f) z, z-x\rangle \leq 0, \quad \forall x \in \Omega .
$$

Proof. Putting $F \equiv 0$, we see that

$$
\left\langle A_{3} x_{n}, y-u_{n}\right\rangle+\frac{1}{r_{n}}\left\langle y-u_{n}, u_{n}-x_{n}\right\rangle \geq 0, \quad \forall y \in C
$$

is equivalent to

$$
\left\langle y-u_{n}, x_{n}-r_{n} A_{3} x_{n}-u_{n}\right\rangle \leq 0, \quad \forall y \in C .
$$


This implies that

$$
u_{n}=P_{C}\left(x_{n}-r_{n} A_{3} x_{n}\right) .
$$

From the proof of Theorem 2.1, we can conclude the desired conclusion immediately. This completes the proof.

Remark 2.5. Corollary 2.4 includes Theorem 3.1 of Yao and Yao [25] as a special case, see [25] for more details.

As some applications of our main results, we can obtain the following results.

Recall that a mapping $T: C \rightarrow C$ is said to be a $k$-strict pseudo-contraction if there exists a constant $k \in[0,1)$ such that

$$
\|T x-T y\|^{2} \leq\|x-y\|^{2}+k\|(I-T) x-(I-T) y\|^{2}, \forall x, y \in C .
$$

Note that the class of $k$-strict pseudo-contractions strictly includes the class of nonexpansive mappings.

Put $A=I-T$, where $T: C \rightarrow C$ is a $k$-strict pseudo-contraction. Then $A$ is $\frac{1-k}{2}$-inverse-strongly monotone; see [1] for more details.

Corollary 2.6. Let $C$ be a nonempty closed convex subset of a Hilbert space $H$ and $F$ be a bifunction from $C \times C$ to $\mathbb{R}$ which satisfies (A1)-(A4). Let $T_{1}: C \rightarrow C$ be a $k_{1}$-inverse-strongly monotone mapping, $T_{2}: C \rightarrow C$ be a $k_{2}$-inverse-strongly monotone mapping, $T_{3}: C \rightarrow C$ be a $k_{3}$-inverse-strongly monotone mapping and $\left\{S_{i}: C \rightarrow C\right\}$ be a family of infinitely nonexpansive mappings. Assume that $\Omega:=F P \cap E P\left(F, I-T_{3}\right) \cap V I \neq \emptyset$, where $F P=$ $\cap_{i=1}^{\infty} F\left(S_{i}\right)$ and $V I=F\left(T_{1}\right) \cap F\left(T_{2}\right)$. Let $f: C \rightarrow C$ be an $\alpha$-contraction. Let $x_{1} \in C$ and $\left\{x_{n}\right\}$ be a sequence generated by

$$
\left\{\begin{array}{l}
F\left(u_{n}, y\right)+\left\langle\left(I-T_{3}\right) x_{n}, y-u_{n}\right\rangle+\frac{1}{r_{n}}\left\langle y-u_{n}, u_{n}-x_{n}\right\rangle \geq 0, \quad \forall y \in C, \\
z_{n}=\left(1-\lambda_{n} u_{n}\right)+\lambda_{n} T_{2} u_{n}, \\
y_{n}=\left(1-\eta_{n} z_{n}\right)+\eta_{n} T_{1} z_{n}, \\
x_{n+1}=\alpha_{n} f\left(x_{n}\right)+\beta_{n} x_{n}+\gamma_{n} W_{n} y_{n}, \quad \forall n \geq 1,
\end{array}\right.
$$

where $\left\{W_{n}: C \rightarrow C\right\}$ is the sequence generated in (1.9), $\left\{\alpha_{n}\right\},\left\{\beta_{n}\right\}$ and $\left\{\gamma_{n}\right\}$ are sequences in $(0,1)$ such that $\alpha_{n}+\beta_{n}+\gamma_{n}=1$ for each $n \geq 1$ and $\left\{r_{n}\right\},\left\{\lambda_{n}\right\}$ and 
$\left\{\eta_{n}\right\}$ are positive number sequences. Assume that the above control sequences satisfy the following restrictions:

(R1) $0<a \leq \eta_{n} \leq b<\left(1-k_{1}\right), 0<a^{\prime} \leq \lambda_{n} \leq b^{\prime}<\left(1-k_{2}\right), 0<\bar{a} \leq r_{n} \leq$ $\bar{b}<\left(1-k_{3}\right), \forall n \geq 1$;

(R2) $\lim _{n \rightarrow \infty} \alpha_{n}=0$ and $\sum_{n=1}^{\infty} \alpha_{n}=\infty$;

(R3) $0<\liminf _{n \rightarrow \infty} \beta_{n} \leq \lim \sup _{n \rightarrow \infty} \beta_{n}<1$;

(R4) $\lim _{n \rightarrow \infty}\left(\lambda_{n}-\lambda_{n+1}\right)=\lim _{n \rightarrow \infty}\left(\eta_{n}-\eta_{n+1}\right)=\lim _{n \rightarrow \infty}\left(r_{n}-r_{n+1}\right)=0$.

Then the sequence $\left\{x_{n}\right\}$ converges strongly to $z \in \Omega$, which solves uniquely the following variational inequality:

$$
\langle(I-f) z, z-x\rangle \leq 0, \quad \forall x \in \Omega .
$$

Proof. Taking $A_{j}=I-T_{j}$, wee see that $A_{j}: C \rightarrow H$ is a $\delta_{j}$-strict pseudocontraction with $\delta_{j}=\frac{1-k_{j}}{2}$ and $F\left(T_{j}\right)=V I\left(C, A_{j}\right)$ for $j=1,2$. From Theorem 2.1, we can obtain the desired conclusion easily. This completes the proof.

\section{Conclusion}

The iterative process (2.1) presented in this paper which can be employed to approximate common elements in the solution set of the generalized equilibrium problem (1.3), in the solution set of the classical variational inequality (1.1) and in the common fixed point set of a family nonexpansive mappings is general. It is of interest to improve the main results presented in this paper to the framework of real Banach spaces.

Acknowledgments. The authors are extremely grateful to the editor and the referees for useful suggestions that improve the contents of the paper.

\section{REFERENCES}

[1] F.E. Browder and W.V. Petryshyn, Construction of fixed points of nonlinear mappings in Hilbert space. J. Math. Anal. Appl., 20 (1967), 197-228.

[2] E. Blum and W. Oettli, From optimization and variational inequalities to equilibrium problems. Math. Stud., 63 (1994), 123-145. 
[3] L.C. Ceng and J.C. Yao, Hybrid viscosity approximation schemes for equilibrium problems and fixed point problems of infinitely many nonexpansive mappings. Appl. Math. Comput., 198 (2008), 729-741.

[4] L.C. Ceng and J.C. Yao, Strong convergence theorems by a relaxed extragradient method for a general system of variational inequalities. Math. Meth. Oper. Res., 67 (2008), 375-390.

[5] S.S. Chang, H.W.J. Lee and C.K. Chan, A new method for solving equilibrium problem fixed point problem and variational inequality problem with application to optimization. Nonlinear Anal., 70 (2009), 3307-3319.

[6] V. Colao, G. Marino and H.K. Xu, An iterative method for finding common solutions of equilibrium and fixed point problems. J. Math. Anal. Appl., 344 (2008), 340-352.

[7] P.L. Combettes and S.A. Hirstoaga, Equilibrium programming in Hilbert spaces. J. Nonlinear Convex Anal., 6 (2005), 117-136.

[8] H. Iiduka and W. Takahashi, Strong convergence theorems for nonexpansive mappings and inverse-strongly monotone mappings. Nonlinear Anal., 61 (2005), 341-350.

[9] A. Moudafi, Weak convergence theorems for nonexpansive mappings and equilibrium problems. J. Nonlinear Convex Anal., 9 (2008), 37-43.

[10] Z. Opial, Weak convergence of the sequence of successive approximations for nonexpansive mappings. Bull. Amer. Math. Soc., 73 (1967), 595-597.

[11] S. Plubtieng and R. Punpaeng, A new iterative method for equilibrium problems and fixed point problems of nonexpansive mappings and monotone mappings. Appl. Math. Comput., 197 (2008), 548-558.

[12] X. Qin, M. Shang and H. Zhou, Strong convergence of a general iterative method for variational inequality problems and fixed point problems in Hilbert spaces. Appl. Math. Comput., 200 (2008), 242-253.

[13] X. Qin, M. Shang and Y. Su, A general iterative method for equilibrium problems and fixed point problems in Hilbert spaces. Nonlinear Anal., 69 (2008), 3897-3909.

[14] X. Qin, M. Shang and Y. Su, Strong convergence of a general iterative algorithm for equilibrium problems and variational inequality problems. Math. Comput. Modelling, 48 (2008), 1033-1046.

[15] X. Qin, Y.J. Cho and S.M. Kang, Convergence theorems of common elements for equilibrium problems and fixed point problems in Banach spaces. J. Comput. Appl. Math., 225 (2009), 20-30.

[16] R.T. Rockafellar, On the maximality of sums of nonlinear monotone operators. Trans. Amer. Math. Soc., 149 (1970), 75-88.

[17] T. Suzuki, Strong convergence of Krasnoselskii and Mann's type sequences for one-parameter nonexpansive semigroups without Bochne integrals. J. Math. Anal. Appl., 305 (2005), 227-239. 
[18] Y. Su, M. Shang and X. Qin, An iterative method of solution for equilibrium and optimization problems. Nonlinear Anal., 69 (2008), 2709-2719.

[19] K. Shimoji and W. Takahashi, Strong convergence to common fixed points of infinite nonexpansive mappings and applications. Taiwanese J. Math., 5 (2001), 387-404.

[20] S. Takahashi and W. Takahashi, Viscosity approximation methods for equilibrium problems and fixed point problems in Hilbert spaces. J. Math. Anal. Appl., 331 (2007), 506-515.

[21] W. Takahashi, Nonlinear Functional Analysis. Yokohama Publishers, Yokohama (2000).

[22] S. Takahashi and W. Takahahsi, Strong convergence theorem of a generalized equilibrium problem and a nonexpansive mapping in a Hilbert space. Nonlinear Anal., 69 (2008), 10251033 .

[23] H.K. Xu, Iterative algorithms for nonlinear operators. J. London Math. Soc., 66 (2002), 240-256.

[24] Y. Yao, M.A. Noor and Y.C. Liou, On iterative methods for equilibrium problems. Nonlinear Anal., 70 (2009), 497-509.

[25] Y. Yao and J.C. Yao, On modified iterative method for nonexpansive mappings and monotone mappings. Appl. Math. Comput., 186 (2007), 1551-1558. 